\title{
Tratamientos químicos de conversión para la protección de magnesio biodegradable en aplicaciones temporales de reparación ósea(*)
}

\author{
M. Carboneras*, L.A. Hernández-Alvarado**, Y.E. Mireles**, L.S. Hernández***, M.C. García-Alonso* y \\ M.L. Escudero*
}

\begin{abstract}
Resumen El presente estudio se desarrolló para mejorar la resistencia a la corrosión del magnesio puro, modificando su superficie mediante recubrimientos de conversión química. Se generaron capas de carbonato y fluoruro por inmersión en soluciones de $\mathrm{NaHCO}_{3}$ al 9 \% y de $\mathrm{HF}$ al 48 \%, respectivamente. La resistencia a la corrosión de las muestras con recubrimiento se evaluó en comparación con la del sustrato desnudo mediante técnicas electroquímicas en una solución fisiológica (PBS). Los resultados obtenidos han mostrado que el recubrimiento de carbonato no es capaz de proteger de la degradación al sustrato de magnesio. Por el contrario, el recubrimiento de fluoruro ha demostrado aumentar, significativamente, la resistencia a la corrosión del magnesio en medio fisiológico. La alta compacidad y adherencia al sustrato metálico de la capa de $\mathrm{MgF}_{2}$ generada mediante este sencillo tratamiento de conversión química confieren al recubrimiento buenas propiedades protectoras.
\end{abstract}

Palabras clave Magnesio; Corrosión; Conversión química; Biomateriales.

\section{Chemical conversion treatments to protect biodegradable magnesium in applications as temporary implants for bone repair}

\begin{abstract}
The present study was developed to improve the corrosion resistance of pure magnesium by applying chemical conversion coatings. Carbonate and fluoride layers were generated by immersion in solutions of $\mathrm{NaHCO}_{3}$ of concentration $9 \mathrm{wt} . \%$ and $\mathrm{HF}$ of concentration $48 \mathrm{wt} . \%$, respectively. Corrosion resistance of the coated samples was evaluated in comparison with that of the uncoated substrate by electrochemical techniques in a physiological solution (PBS). Results have shown that the carbonate coating is not viable to be used for protecting magnesium against corrosion. On the contrary, the fluoride magnesium coating significantly increases the corrosion resistance of magnesium in physiological medium. The high compactness and adherence to the base metal of the $\mathrm{MgF}_{2}$ layer produced by this simple chemical conversion treatment confer the protective properties to the coating.
\end{abstract}

Keywords

Magnesium; Corrosion; Chemical conversion; Biomaterials.

\section{INTRODUCCIÓN}

Los materiales para implantes metálicos convencionales, como aceros inoxidables y aleaciones base titanio, tienen un papel importante en la reparación del tejido del hueso dañado. Si estos implantes permanecen en el organismo por largo tiempo siempre liberan cationes metálicos que pueden dañar la salud. Este problema puede resolverse utilizando implantes biodegradables. Los implantes biodegradables pueden disolverse gradualmente, absorberse o excretarse después de que el hueso se ha reparado. El magnesio y sus aleaciones son materiales biodegradables potenciales debido a su atractivo comportamiento biológi$\mathrm{co}^{[1]}:$ 1) el magnesio se degrada en los fluidos corporales por corrosión; 2) el magnesio no es tóxico y su exceso puede fácilmente ser excretado en la orina; 3 ) el magnesio puede estimular el crecimiento de nuevo tejido en el hueso, haciéndolo particularmente apropiado para aplicaciones ortopédicas; 4) la densidad,

\footnotetext{
(•) Trabajo recibido el día 20 de agosto de 2009 y aceptado en su forma final el día 25 de noviembre de 2009

* Centro Nacional de Investigaciones Metalúrgicas, Madrid, España.

** Facultad de Ciencias Químicas, Universidad Autónoma de San Luis Potosí, San Luis Potosí, México.

*** Instituto de Metalurgia, Universidad Autónoma de San Luis Potosí, San Luis Potosí, México.
} 
módulo elástico y resistencia a la compresión del magnesio son más cercanos a aquellos del hueso que la de los materiales metálicos convencionales empleados en aplicaciones endoprotésicas. Además, el magnesio es necesario para la incorporación del calcio al hueso ${ }^{[2]}$. Debido a estas características, el magnesio y sus aleaciones son potencialmente superiores a cualesquiera otros implantes, metálicos o poliméricos, en reparaciones óseas.

Mientras que la degradabilidad, biocompatibilidad y propiedades mecánicas del magnesio y sus aleaciones son factores deseables para implantes biodegradables, estos materiales sufren de una alta corrosión en los fluidos corporales debido a su contenido en cloruros y otros iones disueltos, no manteniendo su integridad mecánica necesaria durante un periodo suficientemente largo para conseguir una adecuada reparación del hueso. Por ejemplo, la velocidad de corrosión para magnesio con una pureza de $99,9 \%$, en una solución de $\mathrm{NaCl}$ al 3 \% es de 10,5-210 mm por año ${ }^{[3]}$. Es evidente que reducir la velocidad de corrosión de estos materiales en los fluidos fisiológicos, a un valor aceptablemente bajo, es la condición indispensable que debe cumplirse antes de que puedan emplearse como materiales para implantes funcionales temporales y biodegradables.

Para mejorar la resistencia a la corrosión del magnesio y sus aleaciones se han propuesto diferentes alternativas, entre las que cabe destacar los tratamientos de modificación superficial, que incluyen recubrimientos de conversión química ${ }^{[2-8]}$, anodizado $^{[9]}$, electrodepósito de hidroxiapatita ${ }^{[1]}$, entre otros. Los tratamientos para generar recubrimientos de conversión química son conocidos por su bajo coste y simplicidad de operación. En el presente estudio, dos de estos recubrimientos son aplicados sobre sustratos de magnesio puro por simple inmersión a temperatura ambiente con objeto de mejorar su resistencia a la corrosión en medios fisiológicos. Estos recubrimientos se seleccionaron debido a que: a) pueden formarse fácilmente sobre magnesio $\left.{ }^{[10]}, b\right)$ están constituidos por compuestos químicamente inertes, insolubles en muchas soluciones acuosas, que pueden funcionar como recubrimientos tipo ba-

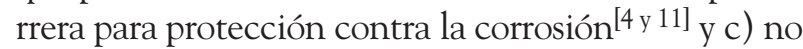
son tóxicos ${ }^{[11-13]}$.

\section{PROCEDIMIENTO EXPERIMENTAL}

Los sustratos sobre los que se aplicaron los recubrimientos fueron muestras de magnesio del 99,8 \% de pureza, obtenido por vía pulvimetalúrgica y, posteriormente, extruido a $420^{\circ} \mathrm{C}$. Previamente a la aplicación de los recubrimientos, las muestras fueron desbastadas con papel de lija de $\mathrm{SiC}$ hasta grado 1.200 $y$, posteriormente, fueron embutidas en una resina poliéster, dejando una superficie de exposición al electrolito de $0,8 \mathrm{~cm}^{2}$. Las condiciones experimentales de aplicación de los recubrimientos de conversión química estudiados se presentan en la tabla I.

La resistencia a la corrosión de las muestras con recubrimiento se evaluó en comparación con la del sustrato desnudo, mediante ensayos electroquímicos, en una solución amortiguadora de fosfato salino (PBS). La composición de la solución de PBS, de $\mathrm{pH}$ 7,4, es la siguiente: $8,0 \mathrm{~g} \mathrm{l}^{-1} \mathrm{NaCl}, 0,2 \mathrm{~g} \mathrm{l}^{-1} \mathrm{KCl}, 0,2$ $\mathrm{g} \mathrm{l}^{-1} \mathrm{KH}_{2} \mathrm{PO}_{4}, 1,15 \mathrm{~g} \mathrm{l}^{-1} \mathrm{Na}_{2} \mathrm{HPO}_{4}$. La osmolaridad y concentraciones de sales inorgánicas hacen que esta solución pueda considerarse una primera aproximación de los fluidos fisiológicos corporales. En este medio corrosivo se pueden observar diferencias de comportamiento frente a la corrosión de los materiales en estudio. Las técnicas experimentales aplicadas en el estudio del comportamiento electroquímico de las muestras de magnesio desnudas y recubiertas fueron: el seguimiento de la evolución con el tiempo, tanto del potencial de corrosión $\left(E_{\text {corr }}\right)$ como de la resistencia a la polarización $\left(R_{p}\right)$ y el trazado de diagramas de impedancia electroquímica con el fin de obtener parámetros cinéticos del sistema en estudio. Las medidas se realizaron por triplicado, utilizando un electrodo de grafito como electrodo auxiliar y un electrodo de referencia de calomelanos saturado (ECS). Tanto los valores de $R_{p}$ como los diagramas de impedancia electroquímica se obtuvieron imponiendo una perturbación al electrodo de trabajo de $\pm 10 \mathrm{mV}$ y registrando la respuesta en corriente del sistema. Todos los ensayos se realizaron en solución de PBS no desaireada y a temperatura ambiente.

\section{Tabla I. Condiciones experimentales de los tratamientos superficiales aplicados al magnesio puro}

Table I. Experimental conditions of the surface treatments applied to pure magnesium

$\begin{array}{ll}\text { Recubrimiento } & \text { Método de } \\ \text { de conversión } & \text { aplicación }\end{array}$

Carbonato Inmersión en solución de $\mathrm{NaHCO} 3$ al $9 \%$ a temperatura ambiente y $\mathrm{pH}=9,0$ durante $20 \mathrm{~h}$ Fluoruro Inmersión en solución de HF al $48 \%$ a temperatura ambiente y $\mathrm{pH}=0,0$ durante $24 \mathrm{~h}$ 
La caracterización de los recubrimientos se llevó a cabo mediante microscopía electrónica de barrido con microanálisis. Los compuestos presentes en el recubrimiento se determinaron mediante difracción de rayos X (DRX) de haz rasante, con ángulo de incidencia de $1^{\circ}$, empleando la radiación Ka del cobre generada a $36 \mathrm{kV} / 30 \mathrm{~mA}$. Las muestras fueron sometidas a un barrido intermitente en el intervalo de $2 \theta$ de $10^{\circ}-70^{\circ}$, con lecturas en $2 \theta$ cada $0,02^{\circ}$ y un intervalo entre lecturas de $3 \mathrm{~s}$.

Finalizados los ensayos de inmersión, los productos de corrosión se caracterizaron mediante espectroscopía de infrarrojo por transformada de Fourier (FTIR).

\section{RESULTADOS Y DISCUSIÓN}

La figura 1 muestra imágenes superficiales de las capas formadas sobre el substrato de magnesio tras los tratamientos de conversión química aplicados. Según se muestra en la figura 1 a), la inmersión en la solución de bicarbonato de sodio forma una capa muy porosa con abundantes grietas sobre la superficie del magnesio. La evolución de hidrógeno en las zonas catódicas pudo contribuir a la formación de poros. Comparativamente, la inmersión de las muestras de magnesio en la solución de ácido fluorhídrico forma películas más uniformes y delgadas (Fig. 1 b)). Como se puede observar, el pequeño espesor de la capa de $\mathrm{MgF}_{2}$ formada permite visualizar las rayas de desbaste del sustrato metálico. Los análisis por DRX corroboraron la presencia de carbonatos y de fluoruros de magnesio en los recubrimientos formados, según se muestra en las figuras 2 a) y 2 b), respectivamente.

La evolución del proceso de degradación de las muestras desnudas y recubiertas con capas de carbonato y fluoruro en medio fisiológico se muestra en la figura 3. Inmediatamente después de la inmersión en PBS, se observó la formación de burbujas de hidrógeno sobre la superficie de las muestras sin recubrimiento y sobre las recubiertas con carbonato, evidenciando así la rapidez del inicio del proceso de corrosión. En la figura 3 a) se puede observar como el valor de $E_{\text {corr }}$ inicial, tanto para el magnesio desnudo como para el recubierto con capa de carbonato se encuentran muy próximos, estando comprendidos entre $-1,550$ y $-1,580 \mathrm{mV}_{\mathrm{ECS}}$. Asimismo, la figura $3 \mathrm{~b}$ ) muestra que los valores de $R_{p}$ para estas muestras son muy similares, siendo inferiores a $4 \times 10^{2} \Omega \cdot \mathrm{cm}^{2}$. Las muestras de magnesio con recubrimiento de fluoruro no mostraban esa evolución, tan rápida, de hidrógeno, exhibiendo potenciales de corrosión más nobles, incrementados en torno a 40-80 mV, así como valores de $R_{p}$ significativamente superiores, en torno a $10^{4} \Omega \cdot \mathrm{cm}^{2}$. A medida que trascurre el tiempo de inmersión el comportamiento de las muestras desnudas y con capa de carbonato es similar, según se extrae del paralelismo entre las curvas que representan la evolución del $E_{\text {corr }}$ y la $R_{p}$ con el tiempo de inmersión (Fig. 3 a) y b), respectivamente.

Finalizado el ensayo de inmersión y extraídas las muestras del medio corrosivo se pudo observar que, tanto las muestras de magnesio desnudas como las recubiertas con capa de carbonato presentaban una corrosión generalizada severa sobre toda la superficie,
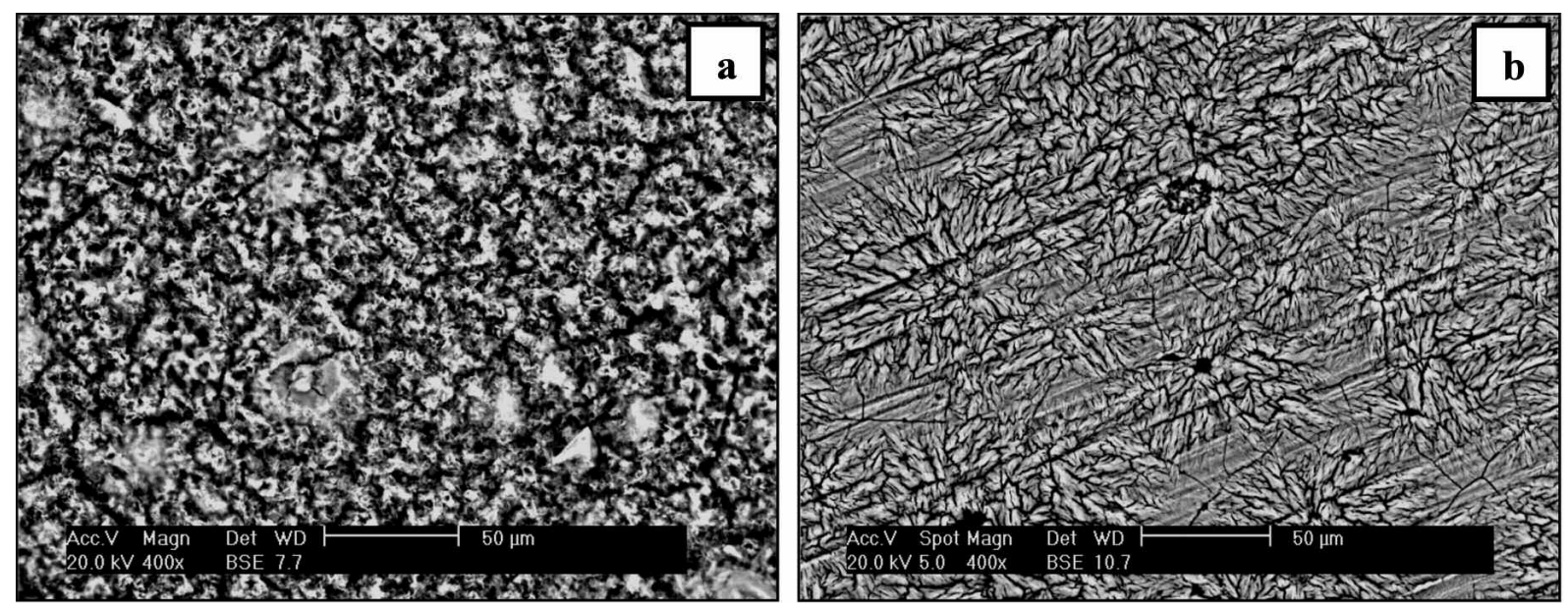

Figura 1. Recubrimientos de conversión química aplicados sobre magnesio puro: a) capa de carbonato y b) capa de fluoruro.

Figure 1. Chemical conversion coatings applied on pure magnesium: a) carbonate layer and b) fluoride layer. 

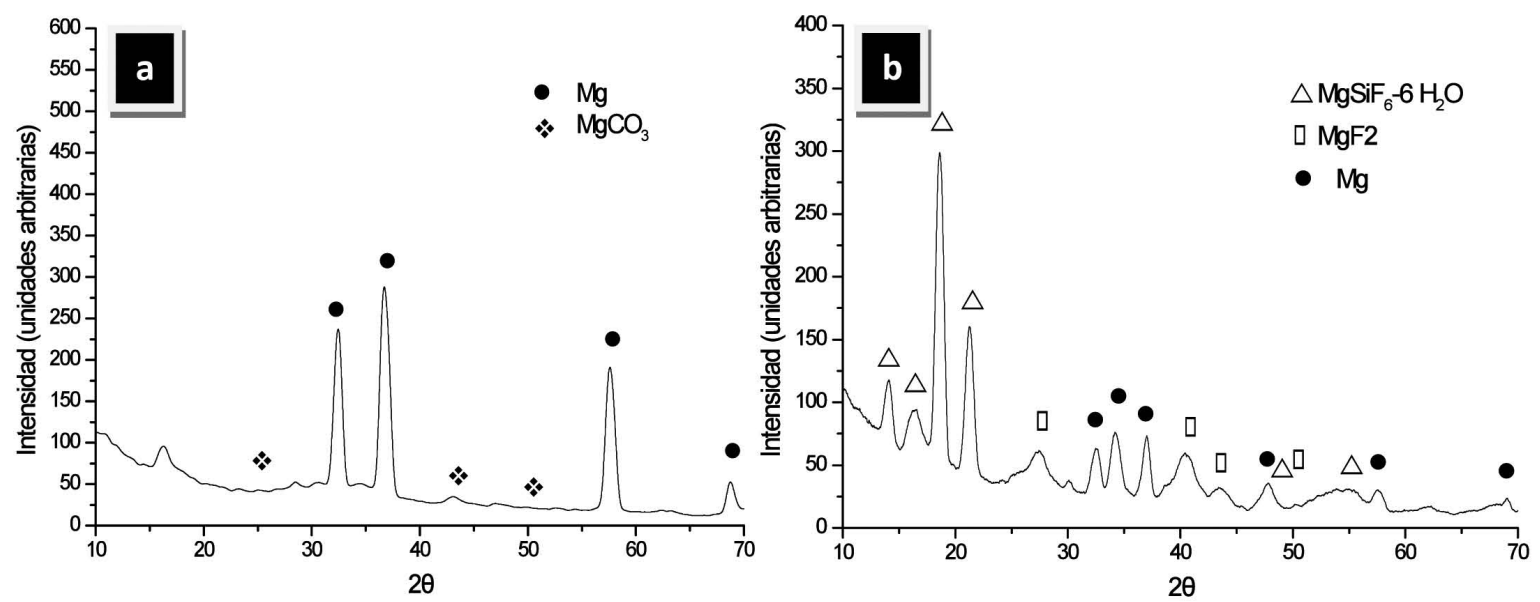

Figura 2. Espectros de difracción de rayos $X$ : a) recubrimiento de carbonato y b) recubrimiento de fluoruro.

Figure 2. X-ray diffraction spectra: a) carbonate layer and b) fluoride layer.
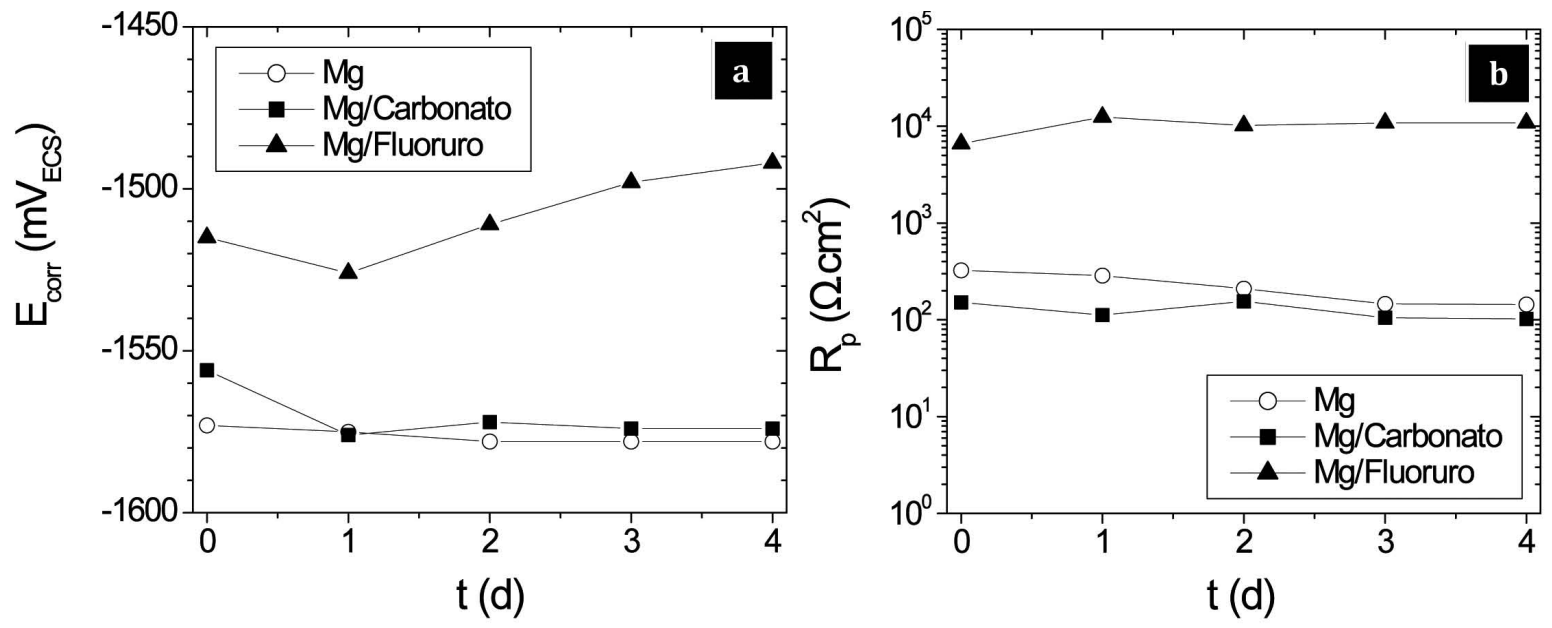

Figura 3. Evolución del potencial de corrosión (a) y de la resistencia a la polarización (b) con el tiempo de inmersión en la solución fisiológica de PBS para el Mg desnudo y recubierto con capas de carbonato y fluoruro.

Figure 3. Evolution of the corrosion potential (a) and the polarization resistance (b) with the immersion time in the physiological solution of PBS for Mg uncoated and coated with layers of carbonate and fluoride.

mostrándose completamente cubiertas con abundantes productos de corrosión de color blanquecino (Fig. 4 a) y b)). Comparativamente, las muestras recubiertas con fluoruro presentaban menor grado de degradación ya que su superficie mostraba menor acumulación de productos de corrosión (Fig. 4 c)).

$\mathrm{El}$ análisis por infrarrojo de los productos de corrosión (Fig. 5) evidencia la presencia de $\mathrm{Mg}(\mathrm{OH})_{2}$ y $\mathrm{MgO}$, así como la precipitación de especies $\left(\mathrm{PO}_{4}\right)^{3}$. Los productos de corrosión que se van formando en presencia de los iones cloruro presentes en el medio fisiológico corrosivo son transformados a través de un proceso de absorción de estos iones, que son fácilmente excretados en situaciones in vivo sin presentar toxicidad ${ }^{[14]}$. Así, estas películas superficiales de óxidos e hidróxidos de magnesio se van transformando en $\mathrm{MgCl}_{2}$, fácilmente soluble, lo que acelera el proceso de corrosión.

Según los resultados obtenidos, el recubrimiento de carbonato no protege al sustrato de magnesio. Por ello, para mayores tiempos de inmersión en el medio agresivo, sólo se realizaron medidas de impedancia 

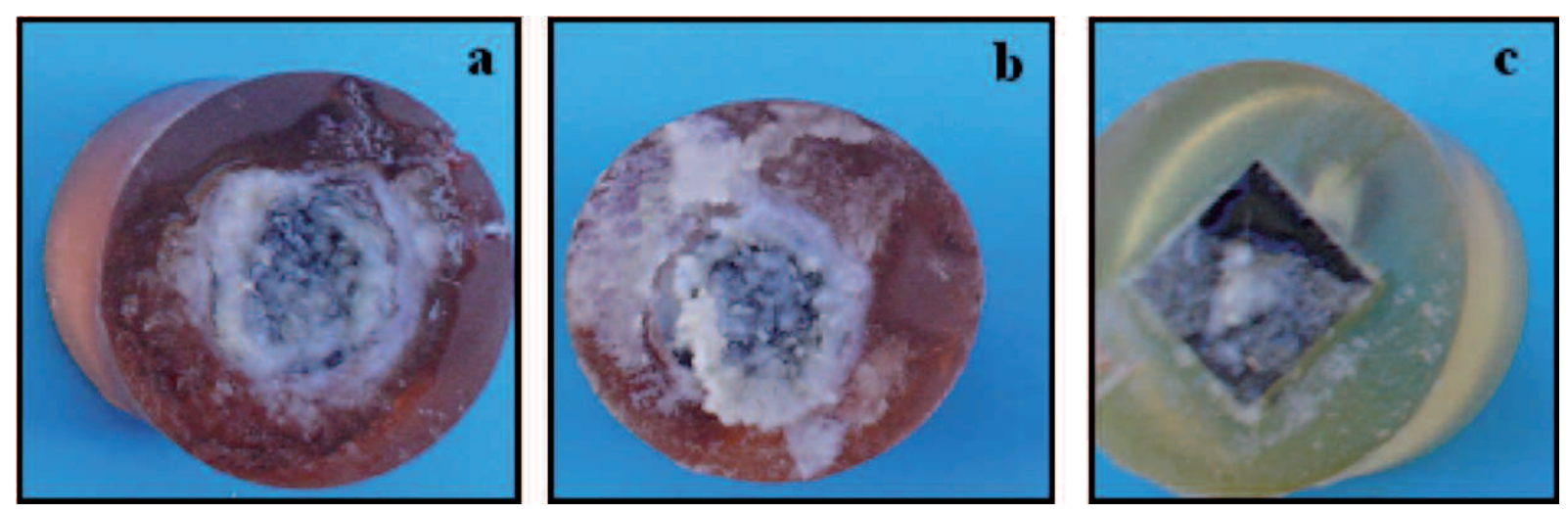

Figura 4. Superficies de las muestras de magnesio después de la inmersión en medio fisiológico (PBS): a) sin recubrimiento, b) con recubrimiento de carbonato y c) con recubrimiento de fluoruro.

Figure 4. Surfaces of magnesium samples after immersion in physiological medium (PBS): a) uncoated, b) carbonate-coated and c) fluoride-coated.

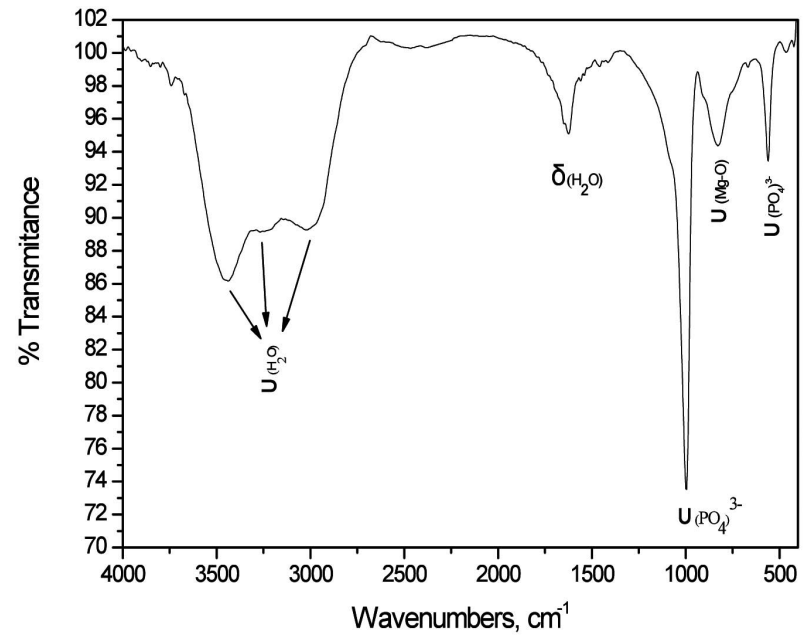

Figura 5. Espectro de IR para la muestra de magnesio con recubrimiento de carbonato sumergida en PBS.

Figure 5. IR spectrum for the carbonate-coated magnesium sample after immersion in PBS solution.

electroquímica para el recubrimiento de fluoruro. Así, la figura 6 muestra los diagramas de Nyquist correspondientes al magnesio desnudo (Fig. 6 a)) y recubierto con capa de fluoruro (Fig. 6 b)) para 1 y 8 días de inmersión en el medio fisiológico de PBS. En la figura 6 a) se puede observar que, transcurrido el primer día de inmersión, el magnesio desnudo presenta un semicírculo a altas y medias frecuencias, seguido de un arco inductivo a bajas frecuencias. Esta forma del diagrama de Nyquist se mantiene con el aumento del tiempo de ensayo hasta 8 días. La figura $6 \mathrm{~b}$ ) muestra el diagrama de Nyquist para las muestras de magnesio recubiertas con una capa de fluoruro. En este caso, se observa la formación de un único semicírculo en todo el intervalo de frecuencias medido $\left(6,4 \times 10^{4} \mathrm{~Hz}-10^{-3} \mathrm{~Hz}\right)$. Fenomenológicamente, el diagrama de impedancia es el mismo para mayores tiempos de inmersión, sin que tampoco se evidencie la presencia de un arco inductivo a bajas frecuencias.

En el estudio del mecanismo de degradación del magnesio y sus aleaciones en medios clorurados, como es el presente caso, el semicírculo obtenido se asigna al proceso de corrosión. En la figura 6 a) se observa que el diámetro de dicho semicírculo para el primer día de inmersión presenta un valor de 600 $\Omega \cdot \mathrm{cm}^{2}$, disminuyendo hasta $80 \Omega \cdot \mathrm{cm}^{2}$, al cabo de 8 días. El valor de este diámetro se asigna a la resistencia a la polarización. Asimismo, se calcularon los valores de la capacidad en el máximo del semicírculo, obteniendo valores comprendidos entre 20 y $40 \times 10^{-6}$ $\mathrm{F} \cdot \mathrm{cm}^{-2}$, valores que se asignan a la capacidad de la doble capa electroquímica ${ }^{[15]}$.

Cuando el magnesio se encuentra protegido con la capa de fluoruro (Fig. 6 b)) se puede observar que el diámetro de los semicírculos es significativamente superior, entre 3 y 4 órdenes de magnitud, mostrando un alto valor de resistencia, en torno a $10^{5} \Omega \cdot \mathrm{cm}^{2}$, que disminuye ligeramente a los 8 días de ensayo. El cálculo de la capacidad en el máximo del semicírculo muestra valores del orden de $10^{-9} \mathrm{~F} \cdot \mathrm{cm}^{-2}$. En este caso, los parámetros obtenidos de los diagramas de impedancia (Fig. 6 b)) no son los parámetros de resistencia de polarización y de la doble capa electroquímica, sino que son los valores de resistencia y capacidad de la capa de fluoruro que está ejerciendo un efecto barrera de protección del magnesio frente al medio corrosivo. Así, la penetración de los iones clo- 

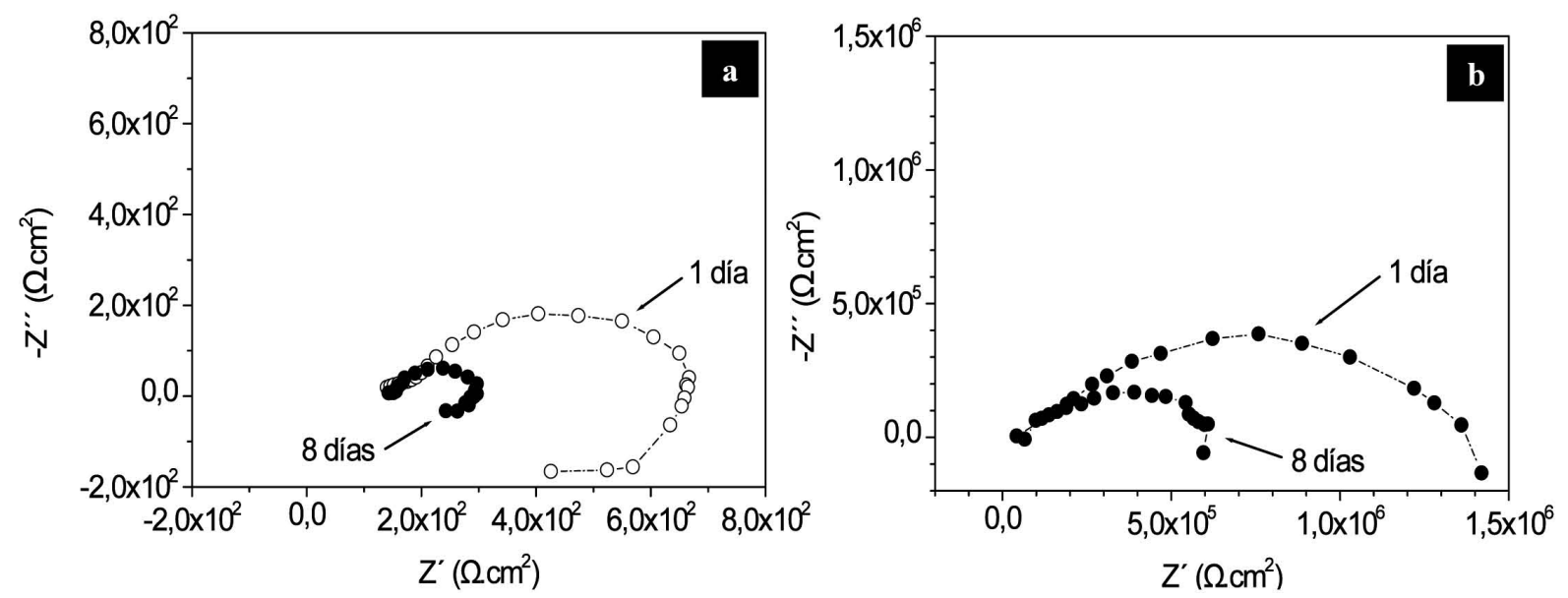

Figura 6. Diagramas de Nyquist para 1 y 8 días de inmersión en PBS: a) magnesio desnudo y b) magnesio con recubrimiento de fluoruro.

Figure 6. Nyquist diagrams for 1 and 8 days of immersion in PBS: a) uncoated magnesium and b) fluoride-coated magnesium.

ruro hacia la superficie metálica se encuentra impedida, de forma que la capa barrera de fluoruro de magnesio, insoluble y no tóxica, frena significativamente el proceso de degradación del magnesio puro.

La figura 7 muestra una imagen de la sección transversal del magnesio protegido mediante el recubrimiento de fluoruro de magnesio. Se puede observar que la capa de conversión química generada presenta una alta compacidad y adherencia al sustrato metálico, características que le confieren unas exce-

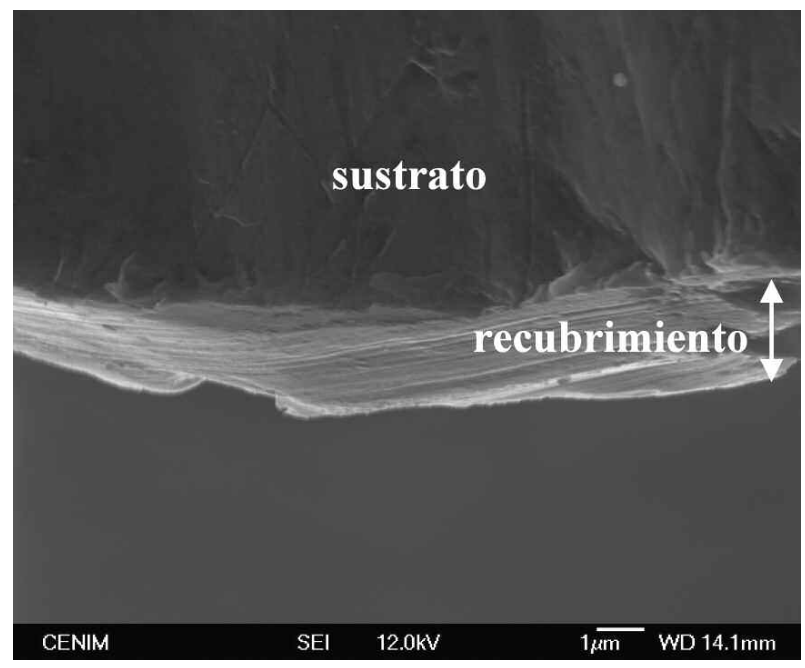

Figura 7. Sección transversal de magnesio puro con recubrimiento de fluoruro de magnesio.

Figure 7. Cross-section image of pure magnesium with fluoride magnesium coating. lentes propiedades de efecto barrera frente a la corrosión del magnesio en medios fisiológicos clorurados como es el PBS. Estos buenos resultados de protección se suman a la excelente biocompatibilidad del magnesio con recubrimiento de fluoruro, ya que recubrimiento y sustrato son reabsorbibles sin producir toxicidad en el medio biológico. De esta manera, este material recubierto biodegradable puede ser considerado como un potencial implante temporal para su uso en reparaciones óseas. Además, dicha biodegradabilidad posibilitará que, una vez que el organismo haya reparado el daño, no sea necesaria una segunda intervención quirúrgica para retirar el material implantado.

\section{CONCLUSIONES}

- El recubrimiento de carbonato formado por inmersión en solución de $\mathrm{NaHCO}_{3}$ al 9 \% no ofrece protección al magnesio frente a la corrosión en medio fisiológico.

- El tratamiento en HF al 48 \% resulta viable en la generación de recubrimientos altamente protectores frente a la degradación del magnesio en medio fisiológico, debiendo ser considerado para futuras aplicaciones del magnesio como implante metálico temporal.

- La alta compacidad y adherencia al sustrato metálico de la capa de $\mathrm{MgF}_{2}$ generada mediante este sencillo tratamiento de conversión química confieren al recubrimiento buenas propiedades protectoras en medio fisiológico. 


\section{Agradecimientos}

Los autores agradecen al MEC la financiación del proyecto con referencia MAT 2008-06719-C03-01. M. Carboneras agradece al CSIC la concesión de un contrato JAE-Doc.

\section{REFERENCIAS}

[1] Y.W. Song, D.Y. Shan y E.H. Han, Mater. Lett. 62 (2008) 3.276-3.279.

[2] Y. Al-Abdullat, S. Tsutsumi, N. Nakajima, M. Ohta, H. Kuwahara y K. Ikeuchi, Mater. Trans. 42 (2001) 1.777-1.780.

[3] L. Li, J. Gao e Y. Wang, Surf. Coat. Tech. 185 (2004) 92-102.

[4] K.Y. Chiu, M.H. Wong, F.T. Cheng y H.C. Man, Surf. Coat. Tech. 202 (2007) 590-598.

[5] L. Jianrui, G. Yina y H. Weidong, Surf. Coat. Tech. 201 (2006) 1.536-1.541.

[6] M.F. Montemor y M.G.S. Ferreira, Electrochim. Acta 52 (2007) 7.486-7.495.
[7] W. Zhou, D. Shan, E. Han y W. Ke, Surf. Coat. Tech. 50 (2008) 329-337.

[8] R. Supplit, T. Koch y U. Schubert, Corros. Sci. 49 (2007) 3.015-3.032.

[9] Y.L. Cheng, H.I. Wu, Z. Chen, H. Wang, Z. Zhang e Y. Wu, Trans. Nonferrous Met. Soc. China 17 (2007) 502-508.

[10] A. Froats, T. K. Aune, D. Hawke, W. Unsworth y J. Hillis, Metals Handbook, Vol. 13, ASM Intl, $9^{\text {th }}$ edition, Ohio, EE. UU., 1987, pp. 740-754.

[11] A. Drynda, T. Hassel, R. Hoehn, A. Perz, F.W. Bach y M. Peuster, J. Biome. Mater. Res. A. (2009) editado "On line" (03.08.2009).

[12] M. Staiger, A. Pietak, J. Huadmai y G. Dias, Biomaterials 27 (2006) 1.728-1.734.

[13] S.F. Lamolle, M. Monjo, M. Rubert, H.J. Haugen, S.P. Lyngstadaas y J.E. Ellingsen, Biomaterials 30 (2009) 736-742.

[14] L. Xu, G. Yu, E. Zhang, F. Pan y K. Yang, J. Biomed. Mater. Res. 83 A (2007) 703-711.

[15] N. Pebere, C. Riera y F. Dabosi, Electrochim. Acta 35 (1990) 555-561. 